Rev. Elev. Méd. Vét. Pays trop., 1976, 29 (1) : 59-66

\title{
Supplémentations minérales, alimentaires et pertes de poids des zébus sahéliens en saison sèche
}

\author{
par H. CALVET $\left(^{*}\right)$, D. FRIOT et I. S. GUEYE
}

\begin{abstract}
RÉSUMÉ
Pour combattre les maladies nutritionnelles existant dans une large région d'élevage du Sénégal, un projet financé par le Fonds d'Aide et de Coopération français (F. A. C.) a été établi qui prévoyait, en particulier, l'installation à proximité du forage de Labgar d'un "Centre de Prévulgarisation des Supplémentations minérales $»$.

Après 5 mois de fonctionnement de ce centre, 1 a été possible de montrer que l'administration régulıère de petites quantités de sels minéraux (apport journalier de 5 à $8 \mathrm{~g}$ de $\mathrm{P}$ ) ou de suppléments protéiques (150 g de MAD par UBT) réduisait les pertes de poids que subissent habituellement les troupeaux au cours de la saison sèche.

Les supplémentations sélectives (celles qui fournissent en quantité minimale les suppléments indispensables) constituent donc un premier pas dans la voie d'une amélioration de la production de l'élevage en zone sahélrenne.
\end{abstract}

\section{INTRODUCTION}

Plus de la moitié du cheptel bovin national est élevé dans la zone sahélienne du Sénégal qui couvre toute la partie septentrionale du pays.

Composés de zébus de race Gobra, groupés en troupeaux comptant en moyenne une centaine de têtes, ces animaux sont entretenus suivant un mode traditionnel très extensif, leur unique source alimentaire étant constituée par les graminées et les arbustes de la savane.

Durant les 50 dernières années, cette région, l'ancien désert du Ferlo, a subi une transformation socio-économique importante du fait de l'implantation de nombreux et importants forages.

L'abreuvement des troupeaux étant désormais assuré durant toute l'année, on a pu observer

$\left(^{*}\right)$ Laboratoire National de l'Elevage et de Médecine vétérinaire. B. P. 2057, Dakar, République du Sénégal. une tendance à la sédentarisation des populations pastorales. Aux grandes transhumances qui permettaient à l'animal de trouver, dans ses pérégrinations à travers des milieux différents, les éléments nutritifs variés indispensables ont succédé des déplacements de faible amplitude entraînant une uniformité de l'alimentation et de ses déficiences. Une des conséquences de cette évolution a été l'apparition de polycarences minérales révélées à l'attention des techniciens de l'élevage par des enzooties meurtrières de botulisme, semant, autour des années 1960, l'inquiétude chez tous les éleveurs de la région. Les recherches entreprises à cette époque, relatées dans plusieurs publications antérieures, ont montré que l'extension du botulisme était favorisée par l'existence d'une maladie nutritionnelle atteignant la plupart des troupeaux, causée essentiellement par une déficience en phosphore, calcium et cuivre.

Après l'application de mesures immédiates, vaccination des troupeaux par les anatoxines 
botuliques $C$ et $D$, i] a été nécessaire d'envisager des méthodes destinées à combattre les carences et à vulgariser l'usage des suppléments minéraux. Ces objectifs ont présidé à l'installation du «Centre de Prévulgarisation des supplémentations minérales de Labgar " dont les premiers mois de fonctionnement ont fourni les éléments de cette note.

L'existence chronique d'une polycarence minérale constitue donc une composante essentielle des élevages extensifs sahéliens qui permet d'expliquer en partie leur comportement et leur productivité relativement faible. Un autre fait important les concernant est lié à la variabilité du milieu naturel dominé par la climatologie tropicale dont la caractéristique bien connue est l'existence d'une courte saison des pluies suivie d'une longue saison sèche. A une brève période d'abondance succède donc une longue époque de disette, la succession dans le temps de ces deux phénomènes induisant chez l'animal une croissance en "dent de scie » caractéristique. Le gain de poids réalisé en période favorable (septembre à décembre) est perdu en grande partie au cours des derniers mois de la saison sèche, et au bout de l'an le bilan pondéral ne se trouve que faiblement positif. Améliorer la production de ces troupeaux consiste donc, de façon toute théorique, à accentuer l'anabolisme et la croissance en période favorable et à diminuer l'intensité du catabolisme et des pertes de poids en saison sèche. Nous verrons comment les supplémentations minérales peuvent contribuer à cet objectif.

La liaison entre nutrition minérale et évolution pondérale ne constitue pas un fait nouveau. Déjà en 1961, BOHMAN et collab. (1) ayant étudié durant 4 ans la croissance de jeunes Hereford entretenus, en zone semi-désertique, sur un ranch du Sud Névada ont montré que si, en hiver, divers compléments alimentaires (avoine ou farine de coton) augmentent le croît de façon significative, en été, (saison chaude et sèche), seule la supplémentation minérale en phosphore produit le même effet.

HARRIS (1953) apporte dans les mêmes conditions des résultats comparables avec $8 \mathrm{~g}$ de $\mathrm{P}$ distribué journellement dans l'eau de boisson. Nous mêmes, au Laboratoire de Dakar en 1972, au cours d'une expérimentation réalisée en étable, avons montré que l'administration en excès de divers suppléments minéraux sur plusieurs lots d'animaux soumis à la même alimentation de base, entraîne un bénéfice pondéral sensible surtout avec le phosphate bicalcique.

Des conclusions comparables vont se dégager des essais, sur le terrain, entrepris à Labgar.

\section{LE CENTRE DE PRÉVULGARISATION DES SUPPLÉMENTATIONS MINÉRALES DE LABGAR}

Pour répondre aux objectifs déjà énoncés (réforme des troubles nutritionnels compliqués de botulısme, amélioration de l'état général des troupeaux et de leur production) un projet intitulé «Valorisation du Cheptel Bovin en zone sylvo-pastorale " a été établi. Ce projet financé par le Fonds d'Aide et de Coopération Français (F. A. C.) comporte 2 parties :

- des enquêtes visant à obtenir une physionomie complète des troupeaux et de la vie socioéconomique des pasteurs de cette région;

- des essais de prévulgarisation des suppléments minéraux entrepris à proxımité du forage de Labgar en vue de démontrer aux propriétaires d'animaux les bénéfices qu'ils sont susceptibles d'obtenir d'un pareil traitement, et d'étudier, sur le terrain, les modalités d'administration les plus favorables.

A cet effet, un Centre, comprenant un certain nombre de bâtiments destinés à loger le personnel et à entreposer le matériel et les produits, a été installé à Labgar, suivant une formule de constructions préfabriquées.

\section{DÉROULEMENT DES OPÉRATIONS}

Un certain nombre de troupeaux sédentaires dans « la zone d'influence » du forage de Labgar sont sélectionnés. Leurs propriétaires s'engagent, en contrepartie du traitement gratuit de leurs animaux, à les soumettre à des pesées de contrôle périodiques et à des prélèvements de sang effectués sur certains individus. L'opération d'identification et la première pesée ont lieu du 14 janvier au 12 février 1972. Les animaux sont marqués à l'oreille et un registre est ouvert, portant le poids, le sexe, l'état de reproduction, la date du premier vêlage, le nombre de veaux 
produits, le nombre d'avortements, le nombre de veaux morts, la filiation et l'origine.

L'effectif de chaque campement est scindé en 2 lots personnalisés par une couleur de marque différente, introduits chacun, tous les soirs, dans des parcs nettement distincts. L'un de ces lots reçoit des suppléments minéraux et l'autre sert de témoin. Cette mise en place se poursuit durant tout un mois et la distribution journalière des suppléments ne commence guère de façon régulière avant la fin mars. Une $2^{\mathrm{e}}$ pesée a lieu en avril 1972. Le court laps de temps entre ces 2 opérations constitue la $1^{\text {re }}$ période. La $3^{c}$ pesée se situe en juin 1972 et marque la fin de la $2^{e}$ période qui couvre ainsi 3 mois.

\section{SUPPLEMENTS DISTRIBUÉS}

Chaque campement est assujetti à un type particulier de traitement différent par la nature du supplément ou par les modalités de distribution.

Pour la supplémentation minérale, trois sortes de phosphates ont été utilisées : le phosphate bicalcique, le polyfos (phosphate aluminocalcique d'origine locale) et un phosphate soluble monosodique a été incorporé à l'eau de boisson à raison de $1 \mathrm{~g}$ par l et distribué journellement à l'aide d'une citerne tractée, dans le campement bénéficiaire.

Les suppléments protéiques comprennent : $\mathrm{du}$ tourteau d'arachide ( $300 \mathrm{~g}$ par UBT), du tourteau d'arachide et des granulés bicalciques, de l'urée contenue dans des pierres à lécher importées composées de 10 p. 100 de $P$, 14,5 p. 100 de $\mathrm{Ca}, 15$ p. 100 de chlorures et 20 p. 100 d'équivalent protéique et de la farıne de riz.

\section{RESULTATS}

Parmi les nombreux résultats obtenus durant la période d'observation (février à juin 1972) qui portent sur la composition des troupeaux, le taux de mortalité des jeunes, le taux de vêlage des femelles, l'évolution de certains paramètres biochimiques, nous ne retiendrons que ceux qui intéressent directement notre sujet : la liaison entre la supplémentation minérale et l'évolution pondérale des animaux.

\subsection{Evolution des poids durant la saison sèche}

Les observations ne couvrent qu'une partie de la saison sèche. En effet, la $3^{e}$ pesée effectuée début juin se situe à plus d'un mois de l'installation normale des pluies. Or, on sait que c'est justement ce dernier mois et les premières 3 semaines accompagnant le renouveau de la végétation qui sont les plus éprouvantes pour les animaux. Les pertes de poids enregistrées constituent donc une large sous-estimation de l'amaigrissement subi habituellement sur la totalité de la saison sèche.

Les résultats sont présentés en fonction des classes d'âge ainsi délimitées :

Classe D comprenant les animaux de 6 mois à 2 ans «faits"

Classe E ceux de 2 à 4 ans

Classe $F$ ceux de 4 à 6 ans

Classe $\mathrm{G}$ ceux de 6 à 10 ans

Classe $\mathrm{H}$ qui regroupe les animaux au-delà de 10 ans.

Le tableau $\mathrm{n}^{\circ}$ I donne l'effectif et les poids moyens enregistrés sur les témoins au cours des 3 pesées.

TABL. N* I-Evolution des poids chez les témoins de février à juin

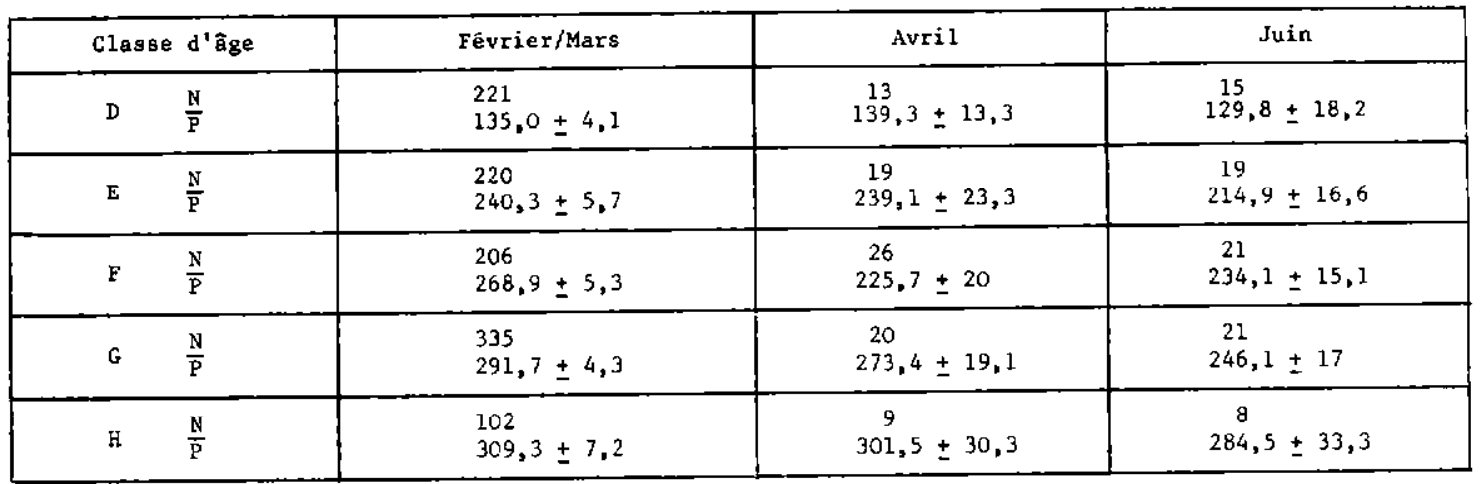


La diminution des effectifs aux pesées d'avril et juin est liée au fait que si la totalité des animaux contrôlés peut être considérée comme témoin lors de la première pesée, avant les supplémentations, il n'en est plus de même après l'institution des traitements. A ce partage entre témoins et traités, se sont ajoutées un nombre important de défections dans les divers troupeaux.

La signification des différences de poids de février à juin a été testée par analyse de variance.

Les valeurs de $F$ pour chaque classe sont présentées dans le tableau suivant:

TABL. $N^{\circ} I I-V a l e u r$ de $F$ pour chaque classe

\begin{tabular}{|c|c|l|}
\hline Classe d'âge & Valeur de F & Degrë de signification \\
\hline D & 0,31 & Non significatif \\
E & 6,28 & Significatif \\
F & 16,67 & Hautement significatif \\
G & 25,05 & Hautement significatif \\
H & 3,18 & Non s1gnificatif \\
\hline
\end{tabular}

La perte de poids que subissent normalement les animaux en saison sèche n'a pu être chiffrée que pour la période allant de février à début juin, qui, comme nous l'avons déjà souligné, ne comprend pas ce mois, le plus critique de tous pour les troupeaux. Les difrérentes classes d'âge sont touchées de façon inégale.

Pour la classe D le pourcentage de perte est de 3,3 p. 100. Les classes E, F, G, H perdent respectivement 10,5 p. $100,14,8$ p. $100,15,6$ p. 100 , 8 p. 100. L'amaigrissement le plus sensible se produit chez les animaux de 6 à 10 ans comptant la majorité des reproducteurs. Ce sont donc ces derniers qui paient le plus lourd tribut à la disette de saison sèche. Sur l'ensemble de l'effectif, la perte est de 11,3 p. 100.

\subsection{Influence des supplémentations minérales sur les pertes de poids de la saison sèche}

Les variations de poids entre les pesées effectuées ont été calculées pour chaque troupeau. Cependant, certaines perturbations étant survenues dans le déroulement du protocole, ce qui ne saurait surprendre quand on connaît le caractère indiscipliné des éleveurs, il a paru nécessaire de présenter 3 interprétations successives. L'une concerne la $1^{\text {re }}$ période, l'autre la $2^{\mathrm{e}}$. La $3^{\mathrm{e}}$ interprétation regroupe les 2 premières phases et couvre ainsi la totalité de l'essai.

\section{Première période}

Les comparaisons concernant les pertes de poids dans les troupeaux témoins et dans les troupeaux traités sont présentées dans le tableau $n^{\circ}$ III. Les valeurs de $F$ donnent le niveau de la signification des différences enregistrées.

S'il n'y a pas de différence significative entre les témoins et l'ensemble des animaux traités, il en existe une dans la comparaison témoinssupplémentation minérale. Les animaux traités, dans ce lot, perdent plus de poids que les témoins.

TABL. NII-Comparaison tëmonns - traitês lère pếriode.

\begin{tabular}{|c|c|c|c|c|c|c|}
\hline & $\mathrm{N}$ & $\mathrm{sx}$ & $S x^{2}$ & $\overline{\mathrm{X}}$ & Valeur de F & Signification \\
\hline Témoins & 25 & -175 & 3981 & -7 & & \\
\hline $\begin{array}{l}\text { Totalité des troupeaux } \\
\text { supplémentés }\end{array}$ & 707 & -11024 & 678626 & -15 & 2,5 & NS \\
\hline $\begin{array}{l}\text { Supplémentation azotée } \\
\text { (stricte) }\end{array}$ & 55 & $-\quad 353$ & 36453 & $-6,4$ & 0,09 & NS \\
\hline $\begin{array}{l}\text { Supplémentation minërale } \\
\text { (stricte) }\end{array}$ & 368 & $-\quad 6747$ & 371487 & $-18,3$ & 4,64 & $\mathrm{~S}$ \\
\hline $\begin{array}{l}\text { Supplémentation minérale } \\
+ \text { azotée }\end{array}$ & 171 & -1530 & 181010 & $-8,9$ & 0,08 & NS \\
\hline $\begin{array}{l}\text { Supplémentation farine } \\
\text { de riz }\end{array}$ & 46 & $-\quad 141$ & 13451 & $-8,9$ & 0,33 & NS \\
\hline $\begin{array}{l}\text { Supplémentation par } \mathrm{PO}_{4} \mathrm{HNa}_{2} \\
\text { dans } 1^{\prime} \text { eau de boisson }\end{array}$ & 67 & - 1983 & 76225 & $-29,6$ & 41,2 & HS \\
\hline
\end{tabular}




\section{Deuxième période}

Alors que la $1^{\text {re }}$ période de supplémentation avait été de courte durée (guère plus d'un mois), la $2^{\circ}$ couvre 3 mois. Comme précédemment, les données concernent les pertes de poids subies par les troupeaux témoins et les divers regroupements d'animaux traités.

Ces résultats font l'objet du tableau $n^{\circ}$ IV.

Il existe donc une différence hautement significative entre les pertes de poids subies par les témoins et l'ensemble des animaux supplémentés.

L'efficacité relative des divers suppléments est en liaison avec les valeurs de $F$ et on peut les ranger suivant ce critère, dans l'ordre décroissant ci-après :
- phosphate monosodique dans l'eau d'abreuvement,

- supplémentation minérale stricte (granulés et pierre à lécher),

- farine de riz,

- supplémentation protéique,

- supplémentation mixte, azotée + minérale.

Les diverses classes d'âge ont profité de façon différente des supplémentations, comme il ressort du tableau $V$.

La supplémentation minérale a eu un effet hautement significatif sur l'évolution des poids chez les animaux âgés de 6 mois à 2 ans et surtout sur les femelles de 6 à 10 ans.

Or, les individus de la classe D maintenus dans des parcs spéciaux n'avaient pas directement

TABL. NIV-Comparaison témoins - traitếs 2e période

\begin{tabular}{|c|c|c|c|c|c|c|}
\hline & $\mathrm{N}$ & $s x$ & $s x^{2}$ & $\bar{x}$ & Valeur de $F$ & Signification \\
\hline Têmoins & 93 & $-\quad 1249$ & 72291 & $-13,4$ & & \\
\hline $\begin{array}{l}\text { Ensemble des troupeaux } \\
\text { supplémentés }\end{array}$ & 675 & 2237 & 498499 & 3,3 & 32,1 & HS \\
\hline $\begin{array}{l}\text { Supplémentation azotée } \\
\text { (stricte) }\end{array}$ & 54 & $\begin{array}{l}-\quad 159\end{array}$ & 27665 & $-2,9$ & 6,5 & s \\
\hline $\begin{array}{l}\text { Supplëmentation minérale } \\
\text { (stricte) }\end{array}$ & 367 & 1990 & 334206 & 5,42 & 31,86 & HS \\
\hline $\begin{array}{l}\text { Supplémentation minérale } \\
\text { et azotée }\end{array}$ & 130 & -1118 & 78800 & $-8,6$ & 2,34 & NS \\
\hline $\begin{array}{l}\text { Supplëmentation à la } \\
\text { farine de riz }\end{array}$ & 56 & 476 & 30336 & 8,5 & 30,24 & HS \\
\hline $\begin{array}{l}\text { Supplëmentation par le } \\
\text { phosphate monosodique } \\
\text { dams l'eau de boisson }\end{array}$ & 76 & 824 & 37400 & 10,8 & 48,9 & HS \\
\hline
\end{tabular}

TABL. $\mathrm{N}^{*} \mathrm{~V}$-Influence de la oupplémentation minérale sur les différentes classes d'âge

\begin{tabular}{|c|c|c|c|c|c|c|c|}
\hline \multicolumn{2}{|c|}{ Classe d'âge } & $\mathbf{N}$ & sX & $s x^{2}$ & $\overline{\mathrm{x}}$ & Valeur de F & Signification \\
\hline \multirow{2}{*}{ D } & Témoins & 18 & -192 & 5484 & $-10,6$ & \multirow{2}{*}{8,3} & \multirow{2}{*}{ HS } \\
\hline & Traitể & 98 & 707 & 68477 & $+7,2$ & & \\
\hline \multirow{2}{*}{$\mathbf{E}$} & Témoins & 24 & -153 & 15977 & $-6,3$ & \multirow{2}{*}{4,6} & \multirow{2}{*}{ s } \\
\hline & Traitéb & 94 & 716 & 84876 & $+7,6$ & & \\
\hline \multirow{2}{*}{$F$} & Témoins & 24 & -256 & 22366 & $-10,6$ & \multirow{2}{*}{2} & \multirow{2}{*}{ NS } \\
\hline & Traitês & 53 & +7 & 52415 & 0,1 & & \\
\hline \multirow{2}{*}{$G$} & Têmoins & 18 & -463 & 22163 & $-25,7$ & \multirow{2}{*}{18,94} & \multirow{2}{*}{ HS } \\
\hline & Traités & 101 & 753 & 99233 & $+7,45$ & & \\
\hline \multirow{2}{*}{$\mathrm{H}$} & Témoins & 9 & -185 & 6361 & $-20,5$ & \multirow{2}{*}{0,76} & \multirow{2}{*}{ NS } \\
\hline & Traitēs & 21 & -193 & 29205 & $-9,2$ & & \\
\hline
\end{tabular}


accès aux suppléments minéraux. La plupart de ces jeunes animaux n'étant pas encore sevrés, le résultat observé ne peut tenir qu'à un effet indirect par la lactation. La supplémentation minérale a donc augmenté la production de lait et les veaux des troupeaux traités ont pu ainsi en tirer profit.

\section{Résultats sur la totalité de l'essai}

Ils portent sur les variations de poids observées entre la première et la dernière pesée, soit durant la totalité de l'observation. Le nombre total des animaux ayant servi à cette analyse, c'est-à-dire le nombre d'animaux retrouvés à la pesée de départ et à celle de juin s'élève à 849 têtes. Malheureusement, et du fait de la défection de nombreux témoins, la répartition entre ceux-ci et les animaux traités est très inégale.

Le tableau no 6 rapporte les résultats.
Tourteau + bicalcique et pierre à

lécher à l'urée. .............F $=9,6$

Cette classification correspond en général aux observations concernant l'aspect général des animaux à l'exception du lot abreuvé sur place par l'eau phosphatée pour lequel les résultats «subjectifs" sont en contradiction avec les calculs statistiques.

En effet, au vu du troupeau concerné et à la satisfaction qu'en éprouvait son propriétaire, on aurait pu classer ce lot parmi les meilleurs. Or, l'étude de la variation de poids sur la totalité de l'observation le met à l'avant dernier rang. Il s'agit en effet du lot qui a perdu le plus de poids durant la $1^{\text {re }}$ période, qui en a rattrapé le plus au cours de la $2^{\text {e}}$, ce qui sur l'ensemble donne le résultat moyen enregistré.

11 'est probable que la longue adaptation des animaux à l'eau acidifiée par le phosphate monosodique n'est pas étrangère à ce comportement.

IABL, $N^{\circ}$ VI-Comparaison tëmoins - traités. Variation de poids sur

la totalité de l'essai

\begin{tabular}{|c|c|c|c|c|c|c|}
\hline & $\mathrm{N}$ & $\mathrm{Sx}$ & $s x^{2}$ & $\overline{\mathrm{x}}$ & Valeur de $F$ & Significaticn \\
\hline Témoins & 27 & $-\quad 857$ & 43097 & $-31,7 \pm 9,8$ & & \\
\hline $\begin{array}{l}\text { Total des animaux } \\
\text { traités }\end{array}$ & 822 & -10516 & 583465 & $-12,8 \pm 1,6$ & 17,10 & HS \\
\hline Supplëmentation azotée & 46 & $-\quad 321$ & 17728 & $-7 \pm 5,5$ & 23,6 & HS \\
\hline $\begin{array}{l}\text { Supplémentation } \\
\text { minêrale }\end{array}$ & 494 & $-\quad 5561$ & 377043 & $-11,3 \pm 2,2$ & 16,88 & HS \\
\hline $\begin{array}{l}\text { Supplémentation azotée } \\
\text { et minérale }\end{array}$ & 167 & -3106 & 121370 & $-18,6 \pm 3,0$ & 9,69 & HS \\
\hline Farine de riz & 38 & $-\quad 203$ & 16889 & $-5,3 \pm 6,8$ & 22 & HS \\
\hline Phosphate soluble & 77 & -1325 & 50635 & $17,2 \pm 4,4$ & 9,85 & HS \\
\hline
\end{tabular}

Sur la totalité de l'essai, les divers traitements ont eu un effet net, comme en témoigne la valeur de $F(17,10)$. En moyenne, l'effectif traité a perdu $19 \mathrm{~kg}$ de moins que les témoins.

L'influence des divers suppléments sur le facteur «diminution des pertes de poids» est donnée par les valeurs de $F$ résultant des comparaisons entre les groupes.

En fonction de ce critère, l'effet des supplémentations dans un ordre décroissant se présente ainsi :

Tourteau arachide. ...........F $=23,6$ Farine de riz . . . . . . . . . . . F $=22$

Supplémentation minérale.......F $=16,8$ Phosphate monosodique........F $=9,8$

\section{Résultats annexes}

Une étude statistique plus poussée a permis d'obtenir un certain nombre d'informations supplémentaires portant en particulier sur la valeur relative des divers produits. Le calcul statistique montre, en premier, que sur l'ensemble de l'essai, la supplémentation minérale donne des effets comparables à la supplémentation azotée ( $\mathrm{F}=1,25 \mathrm{NS}$ ).

$\mathrm{Si}$ on compare par analyse orthogonale l'effet des deux principaux phosphates utilisés (polyfos et bicalcique) on constate qu'il existe une différence hautement significative $(F=27,93)$ entre les pertes de poids entre les lots recevant du polyfos ou du phosphate bicalcique, qui est nettement en faveur du phosphate bicalcique. 
Il existe aussi une différence hautement significative entre les effets des pierres à lécher et des granulés, qui est en faveur des granulés dans le groupe polyfos et en faveur des pierres à lécher dans le groupe bicalcique.

En ce qui concerne les diverses formes de suppléments azotés, on constate que le tourteau pur a été plus efficace que les suppléments azotés et minéraux $(F=12,96)$.

La pierre à lécher à l'urée est aussi peu efficace que le tourteau auquel on adjoint des sels minéraux. Ce fait peut résulter d'un excès d'apport en $P$, car, aux quantités de cet élément contenu dans les granulés, s'ajoutent celles fournies par le tourteau.

\section{DISCUSSION}

Il a paru nécessaire, pour obtenir le maximum d'informations, d'effectuer trois interprétations successives des pertes de poids subies par les troupeaux.

En effet, si les effectifs sont les plus nombreux au cours de la $1^{\text {re }}$ période, la durée d'observation est très courte et les aléas de la mise en place ou les contraintes de l'adaptation entraînent chez certains troupeaux traités des pertes de poids plus élevées que chez les témoins.

La $2^{\mathrm{e}}$ période est beaucoup plus longue, et en conséquence plus fructueuse en résultats, mais ces derniers se trouvent influencés sensiblement par la phase antérieure. En effet, en raison du phénomène de croissance compensatrice, les troupeaux ayant perdu le plus de poids durant l'adaptation sont ceux qui en gagnent le plus par la suite. Il existe donc dans cette $2^{c}$ interprétation une sur-estimation de certains résultats.

L'analyse sur la totalité de l'essai devrait offrir le plus de sécurité et fournir les informations les meilleures sur les effets des divers traitements. Les évolutions pondérales inverses survenues dans les 2 premières périodes ont, en effet, tendance à se neutraliser. Par contre, le nombre des témoins et l'effectif de certains groupes de supplémentés sont par trop réduits.

Ces faits constituent un grave handicap. En effet, les résultats dépendent non seulement de la valeur des suppléments, mais encore du facteur "conduite du troupeau" avec ses 2 composantes : localisation du campement et technicité des pasteurs.
Chaque fois qu'il a été possible de regrouper plusieurs troupeaux soumis au même traitement, le facteur "conduite du troupeau " s'est trouvé plus ou moins " tamponné " et les résultats sont ressortis alors davantage à l'efficacité de la supplémentation. Ce regroupement est la règle en ce qui concerne la supplémentation minérale où l'analyse fait intervenir chaque fois 6 à 10 troupeaux différents, ce qui donne ainsi une grande "crédibilité " aux résultats obtenus.

Les conclusions qui vont être présentées maintenant seront rangées dans un ordre "de sécurité " décroissant, tenant compte des observations précédentes.

Les points suivants peuvent être soulignés :

1) la supplémentation minérale a contribué, de façon manifeste, à diminuer les pertes de poids survenant habituellement en saison sèche, sur le troupeau sahélien. On peut estimer à plus de 8 p. 100 le gain de poids relatif, obtenu chez les troupeaux traités par rapport aux témoins ;

2) la supplémentation par le tourteau d'arachide et la farine de riz paraît produire un effet légèrement supérieur, mais l'observation portant dans chaque cas, sur un seul troupeau, ces résultats présentent peu de sûreté ;

3) les supplémentations mixtes azotées et minérales ont eu un effet moindre que celui des traitements précédents. Mais ces conclusions résultent encore de la surveillance d'un seul troupeau. Dans le cas du troupeau $n^{0} 8$ qui bénéficiait d'un triple avantage, abreuvement sur place, abreuvement journalier, supplémentation par un phosphate soluble, les conclusions statistiques ne correspondent pas aux conclusions subjectives qui auraient conduit à ranger ce lot parmi l'un des plus efficacement traités du projet ;

4) sur la totalité de l'essai, le phosphate bicalcique s'est montré supérieur au "polyfos", phosphate alumino-calcique produit au Sénégal. Cette conclusion corrobore les résultats déjà obtenus au laboratoire et semble justifier la méfiance qu'ont, à l'égard de l'aluminium, la plupart des nutritionnistes du bétall.

\section{CONCLUSION}

La mise en place du Centre de Prévulgarisation minérale de Labgar, au-delà de ses objectifs de vulgarisation des suppléments minéraux, a permis d'obtenir un certain nombre de résultats techniques. 
L'observation, conduite dans un lieu représentatif de la zone sylvo-pastorale, constitue la première expérimentation d'envergure pratiquée au Sénégal, non sans difficultés d'ailleurs, sur le terrain, et en milieu éleveur.

Sa durée a été malheureusement réduite par des conditions climatiques spéciales qui ont contraint les troupeaux à déserter précocement le Ferlo vers des régions privilégiées par une pluviométrie plus abondante. Dans ce cours laps de temps, l'effet des supplémentations instituées n'a pu se marquer que sur un seul paramètre : l'évolution des poids.

La distribution aux troupeaux de très faibles quantités d'éléments minéraux ou de suppléments protéiques a eu pour effet de réduire de façon sensible les pertes de poids subies par les troupeaux qui, chez les témoins, se sont élevées à plus de $10 \mathrm{p} .100$. Ces traitements se sont donc avérés capables, en supprimant les carences, de rétablir les troupeaux dans un état physiologique normal permettant à l'animal de mieux tirer profit du milieu naturel qu'il exploite.

Les supplémentations sélectives constituent donc un premier pas dans la voie d'une plus grande continuité de la production au cours de l'année, indispensable à une exploitation plus intense et plus rationnelle du troupeau sahélien.

\section{SUMMARY}

Mineral supplements and losses of weight

in sahelian cattle during the dry season

To cope with nutritional diseases which may be encountered in a vast extensive cattle breeding zone of Northern Senegal, a FAC sponsered project (Fond d'Aide et Cooperation français) was launched. It consisted particularly in the setting of a prevulgarization center for the study of the influence of mineral supplements

After 5 months of activities of this center, it was demonstrated that daily administration of small amounts of mineral or nitrogen decrease the losses of weight which are usually observed on controls along the dry season.

So the adding of small quantities of supplements to the alimentation is considered as a first step for improvement of extensive cattle production in sahelian areas.

\section{RESUMEN}

\section{Suplementaciones minerales, alimenticias $y$ perdidas de peso de cebues sahelianos en estación seca}

Para luchar contra las enfermedades de la nutrición existiendo en una region vasta de ganaderia en Senegal, se estableció un proyecto financiado por el FAC francès (Fondos de Ayuda y de Cooperación) que preveia, particularmente, ]a instalación cerca del horadamiento de Labgar de un Centro de prevulgarización de los suplementaciones minerales.

Despues de cinco meses de funcionamiento de dicho Centro, fue posible mostrar que la administración regular de pequeñas cantidades de sales minerales (5 à $8 \mathrm{~g}$ de $\mathrm{P}$ por dia) o de suplementos proteicos $(150 \mathrm{~g}$ de MAD por UBT) reducia las perdidas de peso habituales en las manadas durante la estación seca.

Las suplementaciones selectivas (las que dan la cantidad minima de los suplementos indispensables) constituyen el principio de una mejora de la producción de la ganaderia en zona saheliana.

\section{BIBLIOGRAPHIE}

1. BOHMAN (V. R.), MELENDY (H.) et WADE (M. A.). Influence of dietary supplements on growth of beef calves on semidesert range. J. anim. Sci. 1961,20 (3) : 553-557.

2. CALVET $(H$.) et collab. Aphosphorose et botulisme au Sénégal. Rev. Elev. Méd. vét. Pays trop., 1965, 18 (3) : $249-282$.

3. CALVET (H.), FRIOT (D.) et CHAMBON (J.). Influence des supplémentations minérales sur le croît et sur certains témoins biochimiques du métabolisme minéral chez les bovins tropicaux, Rev. Elev. Méd. vét. Pays trop., 1972, 25 (3): 397-408.
4. FRIOT (D.) et CALVET (H.). Etude complémentaire sur les carences minérales rencontrées dans les troupeaux du Nord Sénégal. Rev. Elev. Méd. vét. Pays trop., 1971, 24 (3) : 393-407.

5. HARRIS (L. E.), BUTCHER (J. E.), JAMES (L. F.) et COOK (C. W.). Influence of protein and phosphorus supplements on range cattle production. J. anim. sci., 1957: 1059 (abstract).

6. WARD (H. K.). Supplémentation of beef cows grazing on veld. Rhod J. agric. Res,, 1968, 6 (2): 93-101. 